\title{
Discontinuation Pattern among IUCD Users: A Study at Tertiary Health Care Centre
}

\author{
${ }^{1}$ Roopa Malik, ${ }^{2}$ Vijayata Sangwan, ${ }^{3}$ Smiti Nanda, ${ }^{4}$ Viral Kumar, ${ }^{5}$ Mukesh Kumar
}

\begin{abstract}
Intrauterine contraceptives are the most frequently used reversible family planning method in the world. The method is safe, rapidly reversible, inexpensive, highly effective, long lasting and nonhormonal. These attributes make it unique and desirable for many users. Inspite of all these characters and high initial acceptance, discontinuation remains high. We conducted this study among IUCD users at our department, a tertiary health care centre to determine discontinuation rate and causes for its discontinuation.
\end{abstract}

Keywords: Intrauterine contraceptive device, CuT 380, Contraception, Discontinuation.

How to cite this article: Malik R, Sangwan V, Nanda S, Kumar V, Kumar M. Discontinuation Pattern among IUCD Users: A Study at Tertiary Health Care Centre. J South Asian Feder Obst Gynae 2014;6(1):8-10.

Source of support: Nil

Conflict of interest: None

\section{INTRODUCTION}

The intrauterine contraceptives are the most frequently used reversible family planning method in the world. ${ }^{1}$ Earlier IUCD's were made of inert plastic materials have largely been suspended in modern practice by products which releases copper time to time, modifications in $\mathrm{Cu}$ IUD substantially enhanced their already high efficacy. In 34 randomized control trials involving more than 50,000 woman and 16 different comparisons between intrauterine devices copper IUD were highly effective in preventing pregnancy, with protection lasting up to 12 years for the $\mathrm{CuT}$ 380A. ${ }^{1}$ In India, under Family Planning Program CuT 380A

\footnotetext{
${ }^{1,4}$ Assistant Professor, ${ }^{2,5}$ Senior Resident, ${ }^{3}$ Senior Professor and Head

${ }^{1-3}$ Department of Obstetrics and Gynecology, Post Graduate Institute of Medical Sciences, Rohtak, Haryana, India

${ }^{4}$ Department of Medicine, Post Graduate Institute of Medical Sciences, Rohtak, Haryana, India

${ }^{5}$ Department of Surgery, Post Graduate Institute of Medical Sciences, Rohtak, Haryana, India

Corresponding Author: Roopa Malik, Assistant Professor Department of Obstetrics and Gynecology, Post Graduate Institute of Medical Sciences, Rohtak, Haryana, India, e-mail: drroopa.sangwan@gmail.com
}

is available free of cost in all the government centers. Despite the high popularity of IUD among family planning clients some users discontinue its use for a variety of reasons. We conducted this study among IUD users in our department at a tertiary health care centre to determine discontinuation rate and causes for its discontinuation.

\section{METHODS}

This study was conducted at Pt. BDS PGIMS Rohtak, a tertiary health care centre in Haryana. This was a retrospective study of clients who reported to us from 1st January 2010 to 31 December 2010 for removal of copper T. The analysis done to assess sociodemographic characteristics, side effects, complications, reasons for discontinuation in relation to duration of use, age and parity. All the clients attended family planning clinic for CuT removal were thoroughly interviewed regarding their complaints of bleeding per vaginum, pain abdomen, discharge per vaginum. The clients reported to us for $\mathrm{CuT}$ removal were asked from whom and where they get $\mathrm{CuT}$ inserted in $\mathrm{PHC}, \mathrm{CHC}$ or Private Hospital, whether it was inserted by ANM worker, nurse or a doctor. They were also enquired whether they were counseled about the possible side effects like menorrhagia, pain abdomen, loss of thread, etc. at the time of $\mathrm{CuT}$ insertion. They were also enquired whether they discussed with their husband before $\mathrm{CuT}$ insertion and now for $\mathrm{CuT}$ removal. The females were asked to specify what they meant by 'excessive' bleeding by describing the duration and intensity of blood flow compared with pre IUD insertion menses. Regarding abdominal pain, they were enquired whether they used to have lower abdominal pain before IUD insertion or not and whether it was associated with fever, pain during intercourse and vaginal discharge. They were asked about the interval between the onset of symptom and $\mathrm{CuT}$ insertion. History of use of any other contraceptive method was also asked. The effect of these factors on clients decision of removal of $\mathrm{CuT}$ were assessed. The data analyzed statistically.

\section{RESULTS}

In the year of 2010, a total of 2128 clients attended clinic for contraception. All clients were given 'cafeteria choice' regarding their choice for method of contraception. Thirty percent clients opted for CuT. Simultaneously, we had 
171 clients for removal of $\mathrm{CuT}$ for varying reasons. Majority of the clients $(67.25 \%)$ came for $\mathrm{CuT}$ removal were in the age group of 21 to 30 years. $20.46 \%$ clients were of 31 to 40 years age group. We had 21 clients $(12.28 \%)$ aged more than 40 years (Table 1). Fortyseven $(27.48 \%)$ females opted for CuT removal within 6 months of insertion. Sixteen (9.35\%) clients continued CuT usage for 6 months 1 year. Ninety-two (53.80\%) females used $\mathrm{CuT}$ for 1 to 5 years (Table 2). On analyzing the reasons for CuT removal, 89 (52.04\%) females attended clinic for $\mathrm{CuT}$ removal were wiling for another issue. They used IUCD for child spacing. Nineteen (11.11\%) clients wanted CuT removal for persistent pain abdomen (PID?). Sixteen (9.35\%) had menorrhagia (Table 3$)$. Thirty-six $(20.46 \%)$ clients had onset of symptoms with insertion of $\mathrm{CuT}$ only and got it removed within 3 to 6 months. Persistent pain abdomen and menorrhagia were most common reason for early removal of IUCD. Eighteen females reported to us with missed IUCD 15 clients, among these underwent $\mathrm{CuT}$ removal with office hysteroscope. Three clients, underwent laparotomy for $\mathrm{CuT}$ removal. In one client laparoscopic removal of IUCD was done. ${ }^{6}$ Clients reported to us with failed IUCD at 6 to 8 weeks pregnancy. All of them underwent MTP with CuT removal. Seventy percent among these opted for $\mathrm{CuT}$ as a future contraceptive method. In two clients aged 45 years, husband had expired while in one client husband underwent vasectomy. Five clients attained menopause with $\mathrm{CuT}$ in situ among these one reported to us with pyometera. Her pyometra drainage was done and Lippes loop removed. She had forgotten the loop inserted 25 years back. Fifteen $(8.77 \%)$ clients reported to us with expired duration of $\mathrm{CuT}$. All of them were willing for CuT 380A insertion.

On assessing the associated factors for (Table 4) IUCD removal it was found that females who had $\mathrm{CuT}$ in younger age, $<30$ years, had more incidence of complaints like menorrhagia and pain abdomen and discharge. The cause may be increased sexual activity in the age group. Clients who were counseled well before IUCD insertion about the side effects and underwent insertion by qualified doctors had high chances of continuation. $54.5 \%$ of clients for $\mathrm{CuT}$ removal received no preinsertion counseling. Husband participation was also important, females who had their husbands consent for $\mathrm{CuT}$ insertion bear side effects well and continued IUCD for $\geq 2$ years. $57.03 \%$ clients had no discussion about $\mathrm{CuT}$ removal and insertion with their husband. Level of education also affects the continuation, $70.17 \%$ clients underwent $\mathrm{CuT}$ removal were illiterate. There was history of use of contraceptives in $70 \%$ clients. Seventy percent had history of use of barrier methods, $20 \%$ had history of use of OCP, $6 \%$ had used IUCD and 4\% used natural methods.

\section{DISCUSSION}

CuT IUD first marketed in the early 1970, represent an important contraception option for 150 million women worldwide. The method is safe, rapidly reversible, inexpensive, highly effective, long lasting (up to 20 years for some products) and nonhormonal. These attributes make it unique and desirable for many users. ${ }^{2}$ Inspite of all these characters CuT IUD is also not an ideal contraceptive. It is associated with side effects like menorrhagia, pain in lower abdomen, expulsion, perforation. Different studies reveals different pattern of side effects studies revealed increased bleeding and pain cause upto $15 \%$ of users to have the device removed within the first year. ${ }^{2}$ Hubachec et al in their study revealed dysmenorrhea and bleeding are most important side effects and leads to removal of IUCD in $10 \%$ of women in the 1 st year of use. ${ }^{1,5}$ In our study also persistant pain and excessive bleeding were the main cause of $\mathrm{CuT}$ removal, $20 \%$ clients had these complaints for which they underwent CuT removal within 6 months to 1 year. The most common reason for discontinuation was the desire for pregnancy $52.04 \%$, in other studies it was documented as $57 \% .{ }^{3}$ Other reasons include missed IUCD in $10.52 \%$ clients, menopause in $2.9 \%$ clients, expired Cut in $8.7 \%$ cases. The reason for discontinuation varied significantly with the age of clients, as majority $67.25 \%$ clients were of age group 21 to 30 years. It may be because this is the reproductive span of a

Table 1: Distribution of clients according to age

\begin{tabular}{llll}
\hline S. no. & Age group (yrs) & No. of clients & Clients (\%) \\
\hline 1 & $21-30$ & 115 & 67.25 \\
2 & $31-40$ & 35 & 20.46 \\
3 & $>40$ & 21 & 12.48 \\
\hline
\end{tabular}

Table 2: Distribution of clients according to duration of IU

\begin{tabular}{llll}
\hline S. no. & Duration of IUCD use & No. of clients & Clients (\%) \\
\hline 1 & $0-6$ months & 47 & 27.48 \\
2 & 6 months-1year & 16 & 9.35 \\
3 & $1-5$ years & 92 & 53.8 \\
4 & $>5$ years & 16 & 9.35 \\
\hline
\end{tabular}

Table 3: Reasons for IUCD removal

\begin{tabular}{lllr}
\hline S. no. & $\begin{array}{l}\text { Reason for IUCD } \\
\text { removal }\end{array}$ & No. of clients & Clients (\%) \\
\hline 1 & Persistent pain abdomen & 19 & 11.11 \\
2 & Menorrhagia & 16 & 9.35 \\
3 & Missed IUCD & 18 & 10.52 \\
4 & Unexpected pregnancy & 06 & 3.50 \\
5 & W/F issue & 89 & 52.04 \\
6 & Husband expired & 03 & 1.75 \\
7 & Menopause & 5 & 2.92 \\
8 & Expired duration of CuT & 15 & 8.77 \\
\hline
\end{tabular}




\begin{tabular}{|c|c|c|c|c|c|}
\hline Factors & Total & 3-6 months & 6-12 months & $1-5$ years & $>5$ years \\
\hline \multicolumn{6}{|c|}{ Education } \\
\hline Illiterate & 120 & 29 & 10 & 79 & 02 \\
\hline Literate & 51 & 18 & 06 & 13 & 14 \\
\hline \multicolumn{6}{|c|}{ Discussed with husband } \\
\hline No & 98 & 30 & 06 & 59 & 03 \\
\hline Yes & 73 & 17 & 10 & 33 & 13 \\
\hline \multicolumn{6}{|c|}{ Preinsertion counseling } \\
\hline No & 100 & 32 & 10 & 52 & 06 \\
\hline Yes & 71 & 15 & 06 & 40 & 10 \\
\hline \multicolumn{6}{|c|}{ Previous h/o use of contraceptive } \\
\hline Barrier & 84 & 33 & 10 & 36 & 05 \\
\hline OCP & 24 & 09 & 06 & 09 & 0 \\
\hline IUCD & 07 & 01 & 0 & 04 & 02 \\
\hline Natural & 05 & 04 & 0 & 01 & 0 \\
\hline
\end{tabular}

females life as well as phase of more sexual activity. Other factors like participation of husband for insertion as well as removal, counseling at the time of insertion regarding side effects, education status were also found to be important in decision of $\mathrm{CuT}$ removal, educated clients who had discussed with their husband and counseled well at the time of insertion reported less side effects and continued IUCD longer inspite of side effects than the other clients. In a country like India experiencing a very rapid population growth, it is our duty to counsel females regarding contraception. No doubt CuT IUD has both positive and negative features. The downsides to the utilization of $\mathrm{CuT}$ can be minimized, it requires a trained clinician to insert and proper counseling regarding side effects. WHO advocates use of NSAIDs and hemostatics for control of pain and bleeding associated with IUCD use. ${ }^{4}$ In a country like India with a rapid population growth, contraception is a necessity. Maternal mortality ratio in India is 254 per lakh live birth. ${ }^{9}$ Because of lack of awareness about contraceptive methods to avoid unwanted pregnancy, more MTP by untrained personal has contributed to increase mortality at rural centers. Increased awareness about use of modern contraceptive methods has been shown to reduce unwanted pregnancy, high parity and therefore maternal mortality. ${ }^{6}$ Of all IUCD available, CuT is the oldest method and CuT 380A has been shown to be the best device used in the world today. ${ }^{7,8}$

\section{CONCLUSION}

Intrauterine contraceptive method of family planning was found to be most commonly chosen, highly effective contraceptive method, with high continuation rate at 5 years. The most common cause for IUCD removal was increased bleeding and persistent pain abdomen. So devices that reduce menstrual blood loss and also have longer duration of action like levonorgesterol containing IUCD have a larger scope for continuation. Other countries such as China and Mexico have newer products (different shapes, analgesic releasing, composite materials) that may offer comparable efficacy, yet fewer side effects. The discontinuation rate for the IUCD is high inspite of the high initial acceptability of the method in India. In a country experiencing a very rapid population growth where the prevalence of contraceptive use hardly attains double figures, it is necessary that policy makers double their efforts to ensure an appreciable continuation rate of contraceptive use in general population and IUCD in particular among Indian women.

\section{REFERENCES}

1. Crosignani PG. Intrauterine devices and intrauterine systems. Human Reproduction Update 2008;4(3):197-208.

2. Hubacher D. Side effects from the copper IUD: do they decrease over time. Contraception 2009 May;79(5):356-362.

3. Okunlola MA, Owonikoko KM, Roberts OA, Moihason-Bello ID. Discontinuation pattern among IUCD users at the family planning clinic, University college, Ibadan. J Obstet Gynaecol $2006 \mathrm{Feb} ; 26(2): 152-156$.

4. Alam M, Bradley J, Shabnam F. IUD use and discontinuation in Bangladesh (ACQUIRE Evaluation and Research Studies 2007 Nov; E\&R Study\#8.

5. Hubacher D, Reyes V, Lillo S, et al. Preventing copper intrauterine device removals due to side effects among first time users: randomized trial to study the effect of prophylactic ibuprofen. Hum Reprod 2006;21:1467-1472.

6. Pathak D, Chakraborty B, Goswami S, Adhikari S. Changing trends of maternal mortality: a comparative study. The Journal of Obstetrics and Gynecology of India 2011 March;61(3): 161-165.

7. Obrein PA, Kulier R, Helmerhorst FM, Usher-Patel M, et al. Copper containing framed intrauterine devices for contraception: a systematic review of randomized controlled trials. Contraception 2008 May;77(5):318-327.

8. Grimes DA, Hubacher D, Lopez LM, Schulz KF. Nonsteroidal anti-inflammatory drugs for heavy bleeding or pain associated with intrauterine device use. Cochrane Database Syst Rev 2006; CD006034.

9. Maternal mortality in 2005. Estimates developed by UNICEF, UNFPA, World Bank and WHO, Geneva 2005, WHO 2007. 